\title{
Outstanding Challenges in Recent Open Government Data Initiatives
}

\author{
Usamah A. Algemili* \\ George Washington University, Washington DC, USA. \\ * Corresponding author. Tel.: (202)817-6546; email: gemiliu@gwu.edu \\ Manuscript submitted October 9, 2015; accepted February 17, 2016. \\ doi: 10.17706/ijeeee.2016.6.2.91-102
}

\begin{abstract}
In recent years, we have witnessed increasing interest in government data. Many governments around the world have sensed the value of its passive data sets. These governments started their Open Data policies, yet many countries are on the way converting raw data into useful representation. This paper surveys the previous efforts of Open Data initiatives. It discusses the various challenges that open data projects may encounter during the transformation from passive data sets towards Open Data culture. It reaches out project teams acquiring their practical assessment. Thus, an online form has been distributed among project teams. The questionnaire was developed in alignment with previous literature of data integration challenges. 138 eligible professional participated, and their responds has been analyzed by the researcher. The result section identifies the most critical challenges from project-teams' point-of-view, and the findings show four obstacles that stand out as critical challenges facing project teams. This paper casts on these challenges, and it attempts to indicate the missing gap between current guidelines and practical experience. Accordingly, this paper presents the current infrastructure of Open Data framework followed by additional recommendations that may lead to successful implementation of Open Data development.
\end{abstract}

Key words: Open data, data integration, government data processing, e-government, open government data, project management, project management office.

\section{Introduction}

Open Data defined as "an approach to managing data so that it enables the structured free flow of non-sensitive information to those who have the need or interest in using this information. It allows different types of users to access, organize and use data in ways that make sense to them" [1].

The term Linked Data refers to "data published on the Web in such a way that it is machine-readable, its meaning is explicitly defined, it is linked to other external datasets, and can in turn be linked to from external datasets" [2]. It focuses on publishing Resource Description Framework (RDF) structured data and Uniform Resource Identifiers (URIs) instead of the ontological level or inferencing [3]. Linked data embraces the web of data, and it interlinks decentralized and heterogeneous sources. The web of data aims at replacing isolated data islands with a giant distributed dataset built on the top of web architecture [4], [5].

\subsection{History and Background}

In 2005, Mr. Wing Lam has published his research that identifies 17 barriers to e-government integration (EGI). The research was carried out by Semi-structured interviews that have been conducted with 14 
consultants. Selection criteria of participants were focused on practitioners with significant experience of e-government projects. The research categorizes these barriers into four groups: strategy, technology, policy and organization [6].

This paper discusses the benefits of Open Data, and it casts on potential challenges of Open Data projects. It attempts to extend the previous findings of data integration barriers by Mr. Lam [6]. We survey project teams who work on government data integration projects in order to identify the significant of each barrier from project-teams' point of view. The online questioner addresses an arguably the most critical list of obstacles. We analyze the results based on the replies to the section of the questionnaire followed by some recommendations that may constitute keen OGD (Open Government Data) execution.

\subsection{Open Government Data}

Obviously, the link of open datasets has to offer many benefits [7], and we've witnessed increasing interest in making government data free. Governments recognized Open Data as on approach to increase transparency, advance citizen engagement, economic welfare as well as improving policy-making and public decision-making.

The initiatives of OGD (Open Government Data) are increasing steadily, and this advancement has introduced both opportunities and challenges. For Instance, OGD involves a wide range of stakeholders such as citizens, employees, journalists, researchers, scientists, and engineers. Yet, governments around the world have made more than one million datasets available [8]. These datasets involve various fields such as health, education, transportation networks, budgets, performance and financial reports [9].

Definitely, leaders count on Open Data to bring out many economic advantages. Hence, governmental and semi-governmental agencies keep publishing their data for free [10], [11]. Recent studies show that the reuse of OGD can improve economic growth, accountability and transparency [12], [13]. Moreover, a concrete implementation of OGD policies is expected to promote innovation, social and political change [14]. With open data, investors and citizens are able to know their government's performance that place more interest and trust in public affairs [15].

Open Data may improve political, economic and social values. In 2009, President Obama stated "openness will strengthen our democracy and promote efficiency and effectiveness in government" [16]. Still, many countries rely on OGD to fight corruption, increase transparency and empower civic engagement.

The U.S. government started in May 2009 initiating OGD policy followed by the launch of data.gov website. This policy enforces government agencies to publish high-level data assets for public use. Each agency should publish at least three datasets. As more agencies contribute to the project, the benefits of this policy have risen significantly. Vivek Kundra, former U.S. Chief Information Officer confidently argues that open data stimulate social, political and economic change. He claims that opening data to the public is expected to create economic potential through increased public innovation [17].

Initially, we have witnessed serious initiatives embracing OGD. The US Federal Government and the UK Government released 5,400 datasets as of April 2012; during which they sensed the importance of encouraging public involvement in collect, analyze and use of these sources that improves efficiency and reduces government's spending accordingly. For example, Washington DC invited individuals to implement software applications using open data. At the first call, they received more than 47 applications. The market value of these applications exceeded two million US dollars, not to mention, additional and intangible social impact [18]. New York City holds another annual competition for open data apps, and each year the winner app would cost less than apps that developed by the government offering higher innovation and quality [19].

Open data can create a new potential for economic growth. For instance, the UK Central Government brings in 390 million British Pounds from public sector information. Its supplies cover both personal and 
commercial users [20]. The European Union estimates that earnings from open data reached 140 billion Euros per year. These earnings include direct/indirect contribution beginning from data transactions up to information services [21]. On the other hand, International organizations recognize the benefits of open data. The UN open data handbook provides a long list of benefits including cost efficiency [22]. A 2013 study by McKinsey \& Co. estimates open data could help generate more than $\$ 3$ trillion a year for the global economy [23]. Moreover, a recently conference report by The 3rd International Open Data Conference held in May 2015 confirms the annual potential economic value of open data to reach 3 up to 5 trillion US dollar [24].

Simultaneously, cities and private sector such as Google are deploying data-gathering devices (detectors, sensors, cameras). They are motivated by smart technologies that constitute the recent concept of Smart City. This new infrastructure provides opportunities and challenges that are facing OGD policy makers. The recent paper "Unlocking the Value of Open Data" discusses the applications of Smart City, and it casts on the relation between Open Data and Internet of Things. It confirms the impact of OGD on our cultural, organizational and legal systems [15].

Obviously, public administrations have changed the way data are managed in order to adopt Open Data models, and this change unleashed the ability to publish their own data using government funds. The side effects of this change raised many concerns related to the lack of data quality, incompatible formats, access methods and various semantic interpretations of data [25].

Moreover, OGD may overlook an important segment of its stakeholders who find it hard to utilize OGD due to the lack of user experience and technical knowledge. One solution is to improve data visualizations, which is, at the end, the most favorable approach especially for large datasets of data [26].

Another challenge is that data are frequently offered in different formats, and it is usually missing description or semantics that clearly describe the data. Some administrations solved this problem by presenting the data formally and semantically by Linked Data principles. It combines data set and visualize the results in understandable representation. However, the number of successful integrations has been limited due to many challenges that can be political, social or technical. In most cases, the stakeholders of open data including government agencies find themselves overwhelmed by the amount of unstructured data that makes the benefits of open data services in reasonable doubt.

Open data increases transparency, and administrations have been using tools such as E-voting, surveys on public matters and increase public awareness among citizens. The internal agenda of bureaucracy is efficiency, but governments, in general, have been known as slow in internet adoption. Usually, the main drive for governments is reducing costs. Eventually, e-government delivered many e-services for governments such as service requests, tracking orders, providing information and online payments etc. Accepting online payments has opened the doors for OGD retail as information service. Fig. 1 explains the spheres of e-government and open data [27]. It shows how technology may interact with communication and legal aspects.

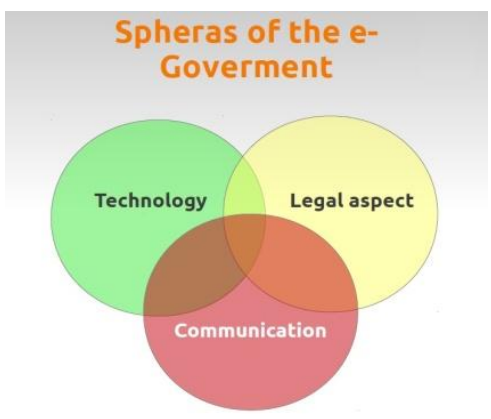

Fig. 1. Spheres of e-government and open data [28]. 
Another benefit of Open Data is that public sector stores information in high-quality formats that reduces the cost of processing. Developers utilize this structured data to develop applications in a timely manner, and then they can focus in other aspects of software engineering such as artificial intelligence [26]. In addition, OGD may provide information about potential markets, and startup companies grow faster in new market areas in which they can see the opportunities. For many IT startups, open data is essential for building their business plan. A survey has been conducted on more than 130 Swedish IT entrepreneurs shows that $82 \%$ believe that open-data access supports their business plan [26].

\section{Research Study}

\subsection{Research Objective}

Our research undertakes the most common obstacles facing OGD projects and it assesses their impact. Study's objective is to identify the outstanding issues that may cause open data projects to be challenged or even failed. It prioritizes Open Data obstacles; then it casts on project challenges that may have been overlooked over the past years. It identifies persistent issues that need serious consideration of IT project management community.

It aims to find the outstanding challenges of open data initiatives followed by quantitative evaluation of each challenge. In reality, barriers impact may vary according to business nature and culture. Nevertheless, this study reports repeating issues that need attention from both executives and project teams.

\subsection{Participants}

The study focuses on the perspective of practical experience rather than theoretical standpoint. Therefore, the participants were selected from practical communities such as IT (Information Technology) teams, project managers, and mid-level executives. The survey targeted more than 2000 IT professional. 153 participants responded to our survey; we excluded 15 participants who answered that they did not work on data integration projects. We analyzed the answers of 138 participants. Chart 1 illustrates participants' distribution among different project levels.

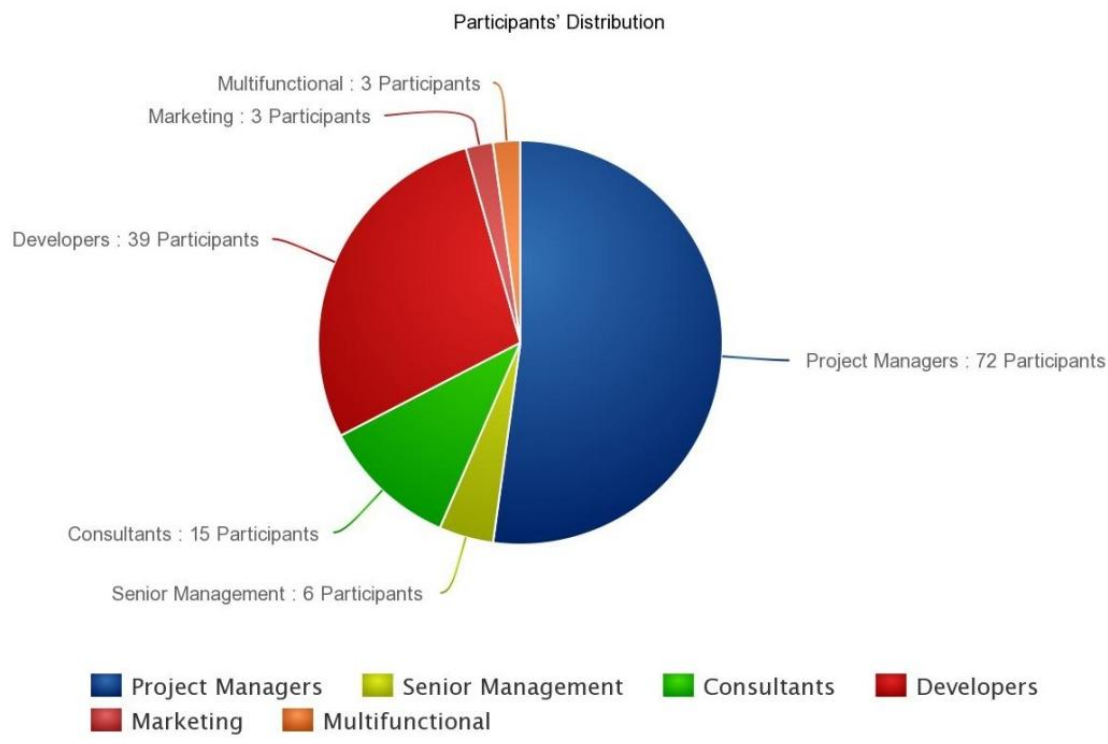

Chart 1. Participants' distribution among project levels.

Chart 2 shows the number of government agencies involved in participants' data integration project. While almost half of the participants have worked only with two agencies, almost $30 \%$ have been working 
in a project that integrate more than four government agencies.

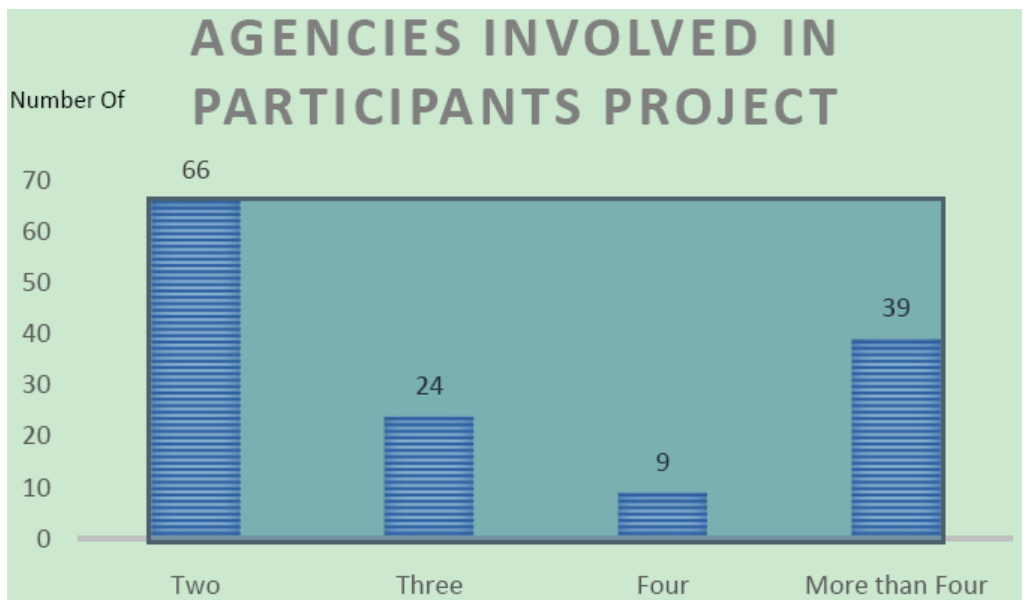

Chart 2. The number of government agencies involved in participants' data integration project.

\subsection{Materials and Procedure}

Our cross-sectional survey was developed within six months collected from developers, project managers, senior managers marketing, sales, and consultants. We used an online form that responsive; web form that easy to reach by any device for quick submit rates, distribute survey link by a private messages or through group applications such as LinkedIn, Twitter, and WhatsApp targeting individuals or professional groups with extensive data integration experience [29].

Questionnaire construction has used sixteen multiple-choice questions grouped into four sections: General, Managerial, Technical and Organizational; response format has used a bounded scale, where the respondent is presented with a range from one to five. The number one represents that the barrier hasn't been a big issue, where the number five expresses very serious difficulty. Simply, we calculated the aggregated score of each barrier's using the number on scale factored by corresponding respondents.

\subsection{Data Analysis and Results}

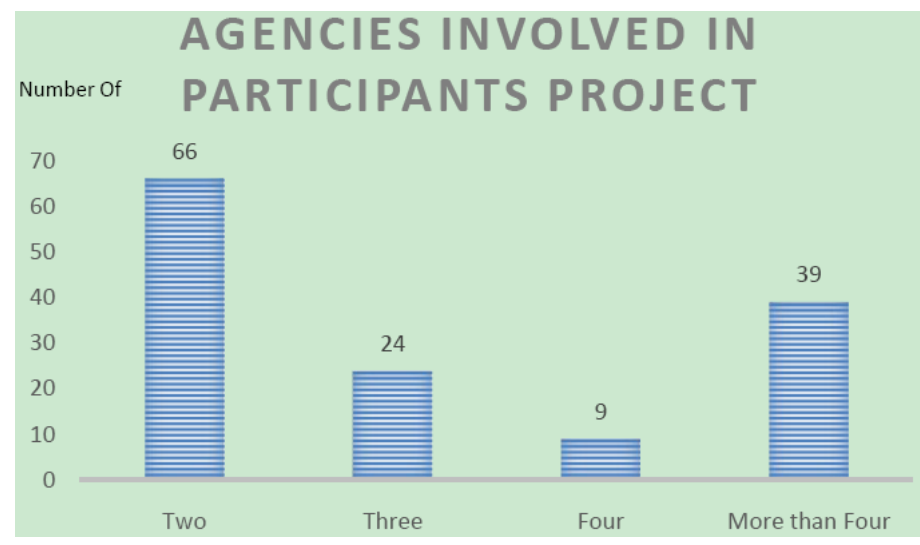

Chart 3. Weighted distribution of each obstacle.

Chart 3 shows the distribution of each obstacle after barriers' weight calculation. We find that four obstacles stand out as critical challenges for project teams. First, incompatible data standards with a score of 513 and weight of $74 \%$. Second, the lack of data ownership and governance data policy with a score of 504 and weight of 73\%. Third, the absence of data processes and data flows with a score of 492 and weight 
of $71 \%$. Finally, the lack of technical expertise with a score of 477 and weight of $66 \%$.

Remarkably, the analysis highlights three challenges that may have been overlooked in the previous literature. Data standards are well addressed by W3C which is a group interested in eGov, but issues such as the lack of data ownership, absence of data flows and the lack of technical expertise need more attention from Open Data stakeholders.

\section{Discussion}

In reality, OGD (Open Government Data) may encounter big challenges in the process of policy making. Mr. Joel Gurin argues that U.S. government should take six legitimate ideas in consideration [30]. These steps would help them build successful Open Data Policy. We cast on four of these ideas. First, a government should encourage agencies to post more information in their open data before any external request. Second, Open Data policy asks government agencies to reveal more data. Nevertheless, data quality is also important, and agencies should improve their data quality reducing possible failures in integration. For Instance, more than forty-five countries have begun open data initiatives by creating user-friendly portals [31]. Third, the continuous improvement of Open Data resources is a very critical process. The US government by its GSA (General Service Administration) has been working on its website "Data.gov", and these efforts have been very useful by providing a central repository of government's open data. In contrast, IT trends are extremely fast and keeping these resources current and up-to-date is a very challenging task. Finally, stakeholders' analysis is one of the key factors in any project's success. Several countries have shown their competency in Open Data projects by applying this concept. Open portals are very useful techniques, and they can be used in both stakeholders' feedback and collective intelligence.

Usually, the primary data is published by e-Government Portals on the internet; it can be unstructured or difficult to reuse as primary data [32]. OGD publishes the raw data in standardized formats that make it reusable.

In the paper "Requirements of an Open Data Based Business Ecosystem" [26], the authors conclude that companies lack the awareness of open data, and they do not know how to use open data sources. Companies may lack sufficient knowledge of license conditions and legal open data policies.

In addition, non-standardized APIs could cause very strong dependency between services and applications. As a result, the promising experience of open data has remained limited at a global scale.

Integration is the most technical challenge related to the use of open data in business. Usually, integration is a part of processing phase. Nowadays, data processing is the most expensive process during open data practice, and integration should have optimization algorithms that would improve processing phase especially if we already know the nature of our raw data [25].

Consequently, the issues of OGD affect us at larger scale, and open data stakeholders include a wide range of actors like government data providers, government data consumers, common citizens, application developers, researchers, and journalists. Fig. 2 illustrates OGD stakeholders and their contribution on open data ecosystems [26], and in the next section we define the role of each actor.

First, Government Data Providers; they consist of people who work in the government, and their primary focus is to make government data available. In most cases, Government Data Providers are responsible of OGD projects within their organization, but they also in charge of improving compatibility inside the government. They would ensure easy access of published data for non-technical people.

Second, Government Data Consumers; they represent people who consume OGD provided by the government, and their core mission is to make the best use of OGD. They usually perform collection, analysis, reporting, feedback and annual proposals. In general, they use Open Data producing sub-products from OGD. In addition, they can store backups which help the users find information when data is not 
available, or when the current visualization is not efficient.

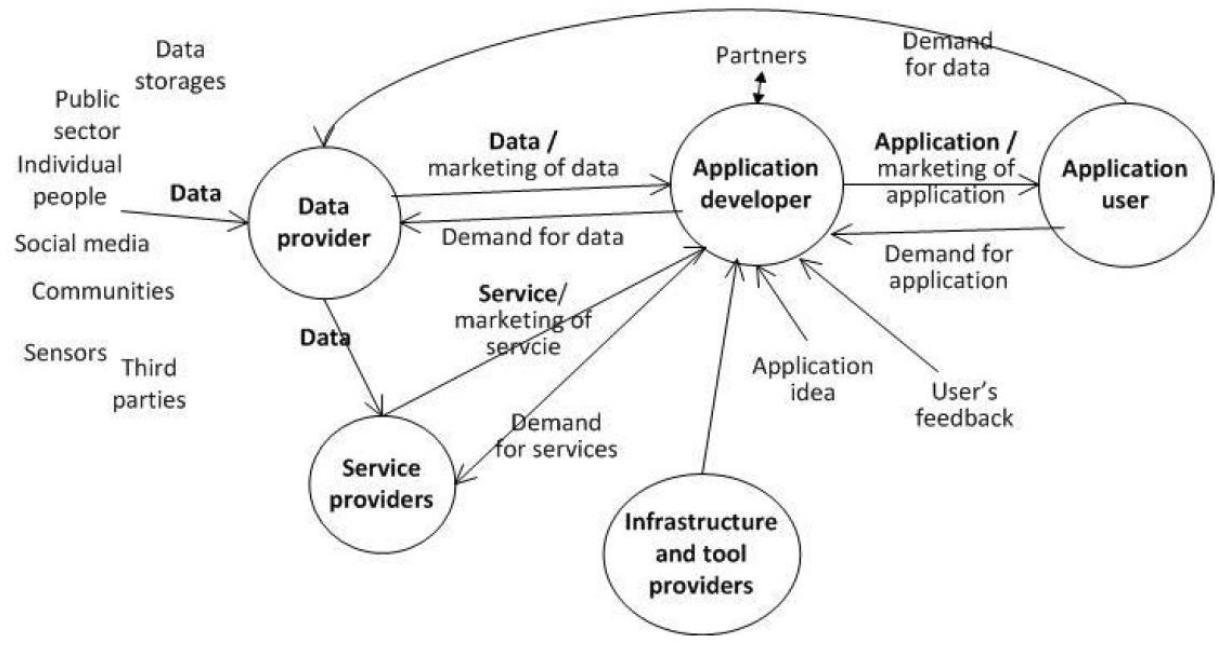

Fig. 2. OGD stakeholders and their contribution to open data ecosystems [26].

Third, Researchers and Journalists who work for non-governmental organizations, and their work focuses on information related to education or health. They use OGD to generate papers and reports. They may use it to advertise certain agendas.

Fourth, Application Developers who have high-level technical skills, and they can create applications that use OGD. They usually have higher-level of goals, yet they are motivated by personal interests. Therefore, governments should maintain the outcomes of OGD application development.

Finally, Common Citizens who are the majority of people, and usually they consume data by a third-party. In general, they use applications and reports. In most cases, they do not have direct connection to OGD [9].

Problems of OGD appear in three different levels creation, exploration, and reuse. In creation stage, OGD involves integrating data from different data sets to produce universal data format. Exploration stage links the data in order to visualize information, and reuse stage modifies the data to achieve further outcomes [9].

We discuss the gap between the desired outcomes and current Open Data initiatives. Most people may lose the benefits of OGD due to lack of technical skills. Therefore, we confirm the importance of OGD visualization services as a critical component of Open Data. Stakeholders need better deliverable to make the best use of OGD.

Public sector collects, stores, presents plenty of information assets, and this information includes economic, geographic and meteorological data. It takes multiple formats, and it stored in different locations. It can be raw data or processed data. For example, population is raw data, while internal processes or public services information such as services waiting times are processed data.

All previous actors are using Open data by different activities, and the analysis of Open Data activities is also important. Zuiderwijk, Janssen, and Jeffery categorize process requirements into twelve sections that should be covered in open data policy [33]: 1). Access, 2). Searching, 3). Navigation, 4). Uploading, 5). Downloading, 6). Data quality, 7). Analysis of data sets, 8). Visualization, 9). Linking and combining data, 10). Collaboration, 11). Support and help and 12). Feedback. The dissection of each activity requirements is out of our scope.

In order to tackle the issues of OGD, project managers may look at open data as an ongoing process rather than a product. It needs continues improvements at all levels from data collection up to visualization. This vision affects OGD requirements; thus OGD infrastructures should provide policies that allow reuse of data. 
In addition, network services should provide services and feedback capabilities free of charge if possible. Linked Data infrastructures on the internet are not enough to serve the whole open data process [26].

A recent paper [34] identifies four classes that institute OGD initiatives. These classes cover both technological and domain aspects. It categorizes OGD as 1. Downloadable files in knowing formats like PDF, CSV, and XML etc. 2. Linked data through APIs or web linking standards. 3. Direct provision through web portals. 4. Indirect provision by the decentralized distribution of data coming from different agencies.

OGD construction consists of multiple issues at many levels; the success of open data relies on the integration among all these issues. In the next discussion, we address these points of interest:

1) Data selection: the organization identifies the data types that can be published without compromising public security and privacy.

2) Data heterogeneity: dealing with unstandardized data format or poor data structure. In some systems, data stored as un-semantic plain text or PDF format.

3) Non-uniform data access: the lack of proper connection protocol dealing with web services.

4) Data security: some components may lack security policies. The level of security measures should be balanced across the framework.

5) Data quality: systems may not have parameters to evaluate the quality of its datasets. These parameters can help indicate possible areas for improvement. In addition, quality assessment points on subsets that are useful for application development.

6) Data processing: all previous issues may impact data processing whereas the interaction among data is very essential. At this stage, data reprocessed by applications to produce services that affect business and market models.

Standardization is an essential aspect of open data. W3C is a group interested in eGov, and they define open data as publishing data in its raw format, machine-readable and full reuse in applications developed by others. W3C offers three standards that may foster open data URI, RDF, and SparQL. URI is a string of characters that used to locate a name or a resource on the internet. RDF is designed to standardize the use of meta-data descriptions in web-based resources. SparQL includes a query language as well as a protocol that access RDF data. It uses datasets that have been published and maintained by a single provider [35].

In the context of OGD development, this paper embraces the ten principles provided by Sunlight Foundation in 2010 [35], and it adds the eleventh property of data ownership. Even though data may be introduced as license-free, keeping ownership records facilitates project team's efforts. This discourse confirms that data ownership arises as a significant factor that can affect both open data and data integration projects. In 2015 Sunlight has expanded its guidelines list to cover three areas and 31 property. However, The following list summarizes these properties followed by the additional attribute of data ownership:

1) Complete: agencies may release all public data that is not subject to privilege, security or privacy limitations.

2) Primary: Data should not be aggregated or modified; it should come from its primary source with high-level of integrity.

3) Timely: Data shall be made available as soon as possible to ensure the fairness of open data opportunity.

4) Accessible: Data must be available and presentable to the various ranges of its stakeholders.

5) Machine Oriented: Data structured reasonably to foster automation.

6) Availability: Data shall be available to anyone without restrictions.

7) Non-exclusive: all parts of its dataset must be unconditionally available, no exclusive segments guaranteed.

8) License-free: Data must be not subject to any Intellectual Property copyright, including trademark, 
trade secret or patent regulations.

9) Security: Data would be subject to the three aspects of Information Security Confidentiality, Availability, and Integrity. In open data policy, Integrity plays very important role in protecting raw data from unauthorized manipulation.

10) Free: Data should be available with no cost, or costs should be minimized as possible.

11) Data Ownership: Data should have ownership property that maintained by clear policy, and it must define access, co-ownership and transformation rights.

In order to utilize OGD, this paper approves three additional elements of open data; these elements are data quality assessment, data accessibility through a universal interface and data correlation and semantic adaptation. These fundamental components would increase the value-added to open data systems followed by valuable feedback to data sources.

\section{Conclusion and Further Work}

Open Data projects need collective and continuous efforts and Open Data policy is definitely the cornerstone, yet other issues rise at both technical and organizational levels. This paper discusses the benefits of OGD and the issues associated with open data projects. Our research assesses the challenges of open data initiatives and government integration projects, in general, followed by recommendation section that covers a wide range of principles. These principles may constitute robust Open Data initiatives. These recommendations take into consideration many aspects that include policy-making, technical implementation, data quality, continuous improvement, stakeholders' analysis and collective intelligence.

Our study highlights data ownership management as an important factor; the impact of data ownership came from its usual fuzziness, in the beginning, for both IT business analysts and project managers.

Open Data would work better in larger scales, and we look forward expanding Open data internationally. Government Leaders across the globe are looking for success stories that foster Open Data initiatives, and by understanding government data integration challenges and its strong interaction with Open Data principles, we may resolve the outstanding challenges of Open Government Data projects by successfully exploiting its evident opportunities.

\section{Appendix}

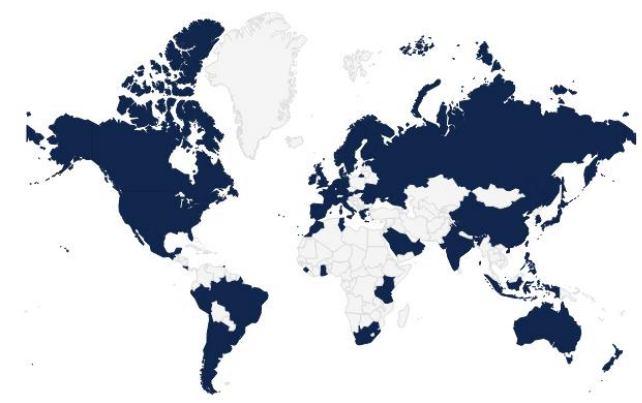

Current deployment of open data internationally (2015) [36].

\begin{tabular}{|l|ll|}
\hline \multicolumn{1}{|c|}{ Principle } & \multicolumn{1}{c|}{ Indicator } \\
\hline Completeness & $-\quad$ Datasets should be as complete as possible and includes \\
& metadata. \\
& $-\quad$ Datasets do not compromise with federal laws on privacy, \\
\hline
\end{tabular}




\begin{tabular}{|c|c|}
\hline & security of privilege. \\
\hline Primacy & 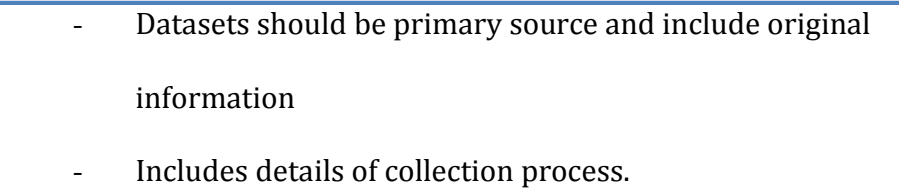 \\
\hline Timeliness & $\begin{array}{l}\text { - Available in timely fashion and be released as quickly as } \\
\text { possible. } \\
\text { - } \quad \text { Release priority depends on the time sensitivity of data. }\end{array}$ \\
\hline Ease of Physical and Electronic Access & $\begin{array}{l}\text { - Datasets should be accessible and easy to be obtained by } \\
\text { any means. } \\
\text { - } \quad \text { Pay attention to the "findability" of datasets. }\end{array}$ \\
\hline Machine Readability & $\begin{array}{l}\text { - Information should be stored in widely-used file formats } \\
\text { and that enable machine processing. } \\
\text { - Documentation related to the format and how to use it is } \\
\text { provided. }\end{array}$ \\
\hline Non-discrimination & $\begin{array}{l}\text { - Any person can access the data at any time and without } \\
\text { having to identify themselves or provide any justification } \\
\text { for doing so. }\end{array}$ \\
\hline Use of Commonly Owned Standards & $\begin{array}{ll} & \text { Used freely available alternative formats. } \\
\text { - } \quad \text { There is no software license needed for access and use. }\end{array}$ \\
\hline Licensing & $\begin{array}{l}\text { - Clear labeling public information as a work of the } \\
\text { government. } \\
\text { - Not subject: copyright, patent, trademark or trade secret } \\
\text { regulation. }\end{array}$ \\
\hline Permanence & Online stickiness - information is available online in perpetuity. \\
\hline Usage Costs & No cost for accessing the data, or cost is as minimum as possible. \\
\hline
\end{tabular}

Sunlight foundation principles (2010) [35]. 


\section{Acknowledgment}

The author wishes to thank all participants in the study for their contribution and valuable feedback.

\section{References}

[1] Cortada, J. W., Nix, V. A., \& Reyes, L. C. (2011). Opening up government: How to unleash the power of information for new economic growth. IBM Institute for Business Value. USA.

[2] Bizer, C., Heath, T., \& Berners-Lee, T. (2009). Linked data - The story so far. International Journal on Semantic Web and Information Systems (IJSWIS), 5(3), 1-22.

[3] Hausenblas, M. (2009). Exploiting linked data to build web applications. IEEE Internet Comput., 13(4), 68-73.

[4] Hausenblas, M., \& Karnstedt, M. (2010). Understanding linked open data as a web-scale database. Proceedings of the 2nd International Conference on Advances in Databases Knowledge and Data Applications, Menuires, France (pp. 56-61).

[5] Heath, T. (2008). How will we interact with the web of data? IEEE Internet Comput., 12(5), 88-91.

[6] Lam, W. (2005). Barriers to e-government integration. Journal of Enterprise Information Management 18(5), 511-530.

[7] Hoxha, J., \& Brahaj, A. (2011). Open government data on the web: A semantic approach. 2011 Proceedings of International Conference on, Emerging Intelligent Data and Web Technologies (EIDWT) (p. 107).

[8] Graves, A., \& James, H. (2013). Visualization tools for open government data. Proceedings of the 14th Annual International Conference on Digital Government Research.

[9] Rozell, E., Zheng, J., Shi, Y., Lebo, T., Erickson, J., Shinavier, J., Williams, G. T., \& Graves, A. IOGDS: International open government dataset search. Retrieved from the website: http://logd.tw.rpi.edu/demo/international_dataset_catalog_search

[10] McDermott, P. (2010). Building open government. Government Information Quarterly, 27, 401-413.

[11] Geiger, C. P., \& Lucke, J. V. (2012). Open government and (linked) (open) (government) (data). Journal of e-Democracy and Open Government, 4, 265-278.

[12] Zhang, J., Dawes, S., \& Sarkis, J. (2005). Exploring stakeholders' expectations of the benefits and barriers of e-government knowledge sharing. Journal of Enterprise Information Management, 18, 548-567.

[13] Janssen, M., Charalabidis, Y., \& Zuiderwijk, A. (2012). Benefits, adoption barriers and myths of open data and open government. Information Systems Management, 29, 258-268.

[14] Sayogo, Djoko, S., \& Theresa, A. P. (2013). Understanding smart data disclosure policy success: The case of Green Button. Proceedings of the 14th Annual International Conference on Digital Government Research.

[15] Xavi, M.-B., et al. (2013). Unlocking the value of open data with a process-based information platform. Proceedings of 2013 IEEE 15th Conference on Business Informatics (CBI).

[16] Obama, B. Transparency and Open Government. Retrieved from the website: https://www.whitehouse.gov/the_press_office/TransparencyandOpenGovernment

[17] Kundra, V. (2011). Digital fuel of the 21st century: Innovation through open data and the network effect. Retrieved from the website: http://shorensteincenter.org/2012/01/digital-fuel-of-the-1stcentury-innovation-through-open-data-a nd-the-networkeffect/

[18] Apps for democracy. Retrieved from the website: http://www.appsfordemocracy.org/.

[19] Big Apps NYC. Retrieved from the website: http://nycbigapps.com/ 
[20] Newbury, D., Bently, L., \& Pollock, R. (2008). Models of public sector information provision via trading funds. UK Department of Business, Enterprise and Regulatory Reform.

[21] Vickery, G. (2011). Review of recent studies on PSI re-use and related market developments. Information Economics. France.

[22] Davies, T. (2010). Open data, democracy and public sector reform: A look at open government data use from Data.Gov.UK.

[23] Barne, D. (2014). What can open data entrepreneurs do for development? Voices.

[24] Report. International Open Data Conference 2015.

[25] Zuiderwijk, A., et al. (2012). Linking open data: Challenges and solutions. Proceedings of the 13th Annual International Conference on Digital Government Research.

[26] Immonen, A., Palviainen, M., \& Ovaska, E. (2014). Requirements of an open data based business ecosystem. Access, 2, 88-103.

[27] Lakomaa, E., \& Jan K. (2013). Open data as a foundation for innovation-the enabling effect of free public sector information for entrepreneurs.

[28] Maurizio, N. Open Data Challenges and Opportunities. Online Presentation.

[29] Algemili, U. Government data integration. Retrieved from the website: https://docs.google.com/forms/d/1sdzF11LVsGRgFYWNzvjdA4gNXQFomToKxwm-1rKm2wA/viewfor $\mathrm{m}$

[30] Joel, G. Information week. Retrieved from the website: http://www.informationweek.com/author-bio.asp?author_id=1117.02/01/2014

[31] Open Government. Data.gov. Datagov. Datagov. Web. (2015). Retrieved from the website: https://www.data.gov/open-gov/

[32] Berners-Lee, T., Hendler, J., \& Lassila, O. (2001). The semantic web. Scientific American, 284(5), 34-43.

[33] Zuiderwijk, A., Janssen, M., \& Jeffery, K. (2013). Towards an e-infrastructure to support the provision and use of open data. Proceedings of Conference for e-Democracy and Open Government. Donau, Austria.

[34] Kalampokis, E., Efthimios, T., \& Konstantinos, T. (2013). On publishing linked open government data. Proceedings of the 17th Panhellenic Conference on Informatics.

[35] Ten Principles for Opening up Government Information — Sunlight Foundation: 2010. Retrieved from the website: http://sunlightfoundation.com/policy/documents/ten-open-data-principles/

[36] Open Government. (2015). Data.gov. Datagov. Retrieved from the website: https://www.data.gov/open-gov/

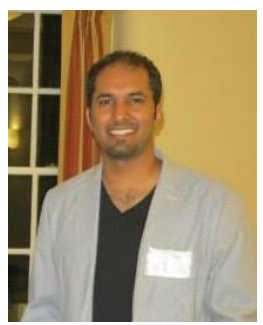

Usamah Algemili is a Ph.D. candidate at the Department of Computer Science at The George Washington University holding project management professional (PMP®) designation from Project Management Institute. He received his BSc degree in computer science from King Saud University, Saudi Arabia, in 2004. Algemili earned his master degree in computer science from George Washington University, Washington, DC, USA in 2012. His research interests are big data processing, information retrieval systems, and open data integration. 